Wacholder, N., Venuti, J., Krauthammer, M., \& Molholt, P. (2000). Accessing and browsing 3D anatomical images with a navigational ontology..

11th ASIS SIG/CR Classification Research Workshop, 75-85. doi:10.7152/acro.v11i1.12770

\title{
Accessing and Browsing 3D Anatomical Images with a Navigational Ontology
}

\author{
Nina Wacholder $\mathrm{PhD}^{1,2}$, Judith Venuti $\mathrm{PhD}^{1,3}$, Michael Krauthammer $\mathrm{MD}^{4}$, \\ and Pat Molholt $\mathrm{PhD}^{1}$ \\ Columbia University \\ ${ }^{1}$ Office of Scholarly Resources; ${ }^{2}$ Center for Research on Information Access; \\ ${ }^{3}$ Department of Anatomy and Cell Biology; ${ }^{4}$ Department of Medical Informatics \\ nina@cs.columbia.edu
}

\begin{abstract}
The problem that our research addresses is the lack of a comprehensive, universally useful system for navigating 3D images of anatomical structures. In this paper we discuss the organization of anatomical information in a navigational ontology, a knowledge representation formalism that supports intelligent browsing of $3 \mathrm{D}$ anatomical images. For the purposes of this project, 'intelligent' means that the computer system behaves as if it had accurate knowledge of human anatomy consistent with that of a trained anatomist (though not necessarily as complete). To give a simple example, if the user asks to see the component structures of the urinary system, the system will return to the user either a list of structures and/or a model of them, just as an anatomy instructor might do.

The Vesalius Anatomy Browser provides an interface for navigating 3D anatomical images in which anatomical images are linked to a hierarchical representation of conceptual information that corresponds directly to the images displayed on the screen. The association of the concepts with images makes possible simultaneous visual exploration of anatomical information via word and image.
\end{abstract}

\section{Overview}

The Vesalius ${ }^{\mathrm{TM}}$ Anatomy Browser (VAB), is a web-based software tool (Krauthammer 1999, http://www.cpmc.columbia.edu/projects/vesalius) that accesses information in the Vesalius ${ }^{\mathrm{TM}}$ Knowledge Base (to be discussed below) whose two-part display is organized around a focus concept, in this case the bladder. 


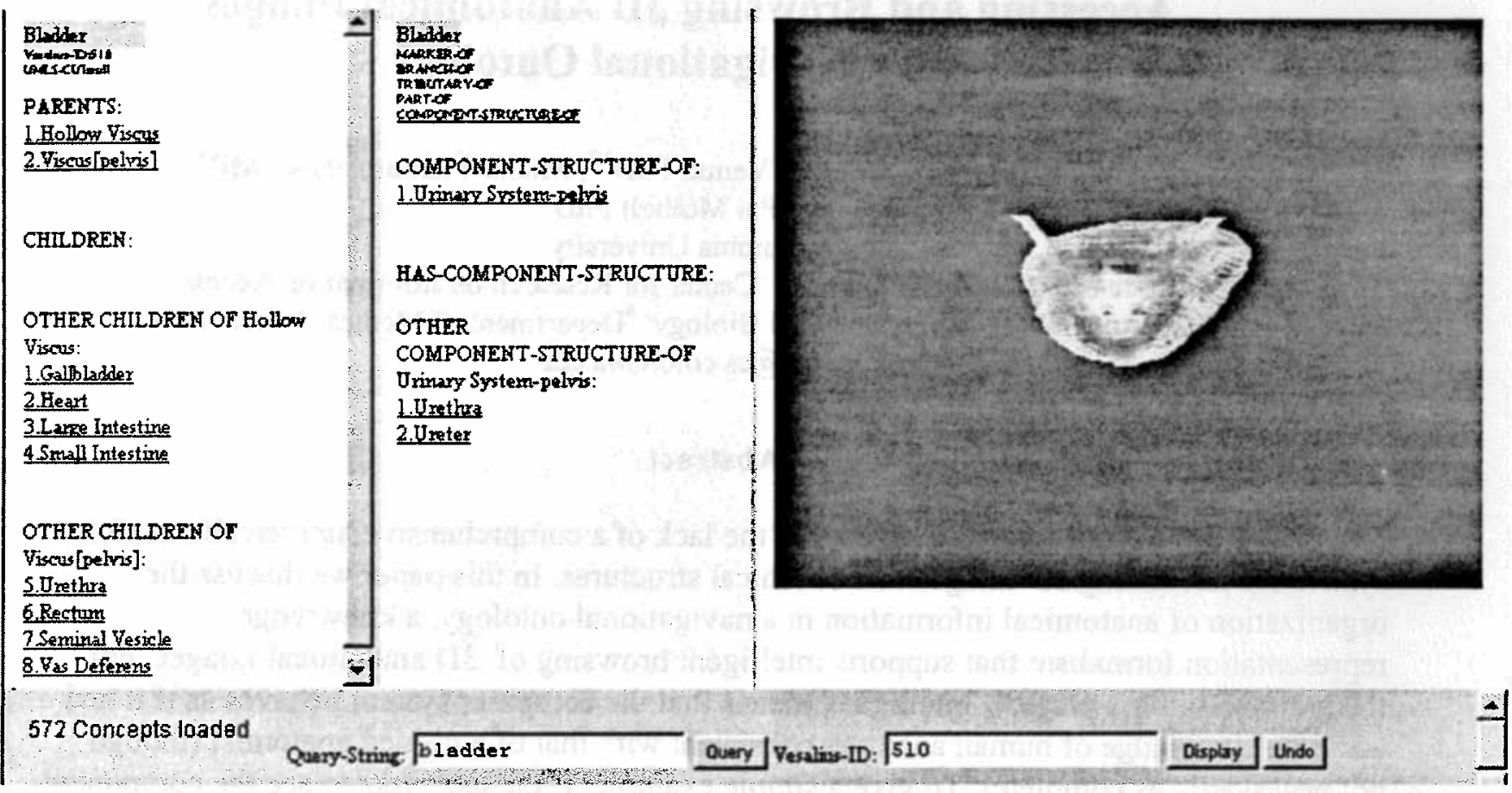

Figure 1: Screenshot from the Vesalius Anatomy Browser

In one frame is an image of the bladder reconstructed from the Visible Human data (Ackerman 1995; Imielinska et al. 1998; Imielinska et al. 1996; Wacholder et al. (to appear)); this image can be rotated 360 degrees along the $Y$ axis. In the other frame, the name of the focus concept is highlighted, and anatomical entities in specific relationships to it are displayed in a viewable fragment of the ontology that we call the concept space. The concept space is a kind of concept mapping [Novak and Musonda, 1991], in which the meaningful relationships among concepts are represented graphically. Concept mapping has been shown to be useful in education in general and in medical education in particular [Horton et al. 1993; Pinto and Zeitz 1997; Jonassen et al. 1997). The relationship between the concept space of the navigational ontology and concept mapping are discussed in more detail below.

In the $\mathrm{VAB}$ concept space, the focus concept is shown to be related to other anatomical structures in ways that are specified in the navigational ontology. For example, the bladder is one of the pelvic viscera, as are the urethra, rectum, seminal vesicle and vas deferens. The bladder is also a hollow viscus, as are the gall bladder, heart, large intestine and small intestine. The bladder is a component of the pelvic urinary system, as are the urethra and ureter. Clicking on any anatomical concept makes it the focus concept; other anatomical structures related to it are displayed in relation to the new focus concept.

The premise behind the organization of the display is that a user interested in looking at a given anatomical entity is likely to want to view next one or more anatomical structures related to it in some anatomically significant way, whether spatially, conceptually or functionally. After looking at an image of the bladder, the user might want to see the entire pelvic urinary system, of which the bladder is part, or the trigone, a smooth area of the bladder's epithelial lining. By displaying related structures rather than an alphabetized index 
Wacholder, N., Venuti, J., Krauthammer, M., \& Molholt, P. (2000). Accessing and browsing 3D anatomical images with a navigational ontology..

11th ASIS SIG/CR Classification Research Workshop, 75-85. doi:10.7152/acro.v11i1.12770

of all of the structures in the system, the display serves as a context-sensitive navigational system for anatomical images. We plan to use the navigational ontology as the basis for two applications which require visual exploration of anatomical images:

- a study tool which we call a 'curriculum-driven ontology'. This curriculum ontology presents anatomical concepts which students are responsible for mastering in a particular unit or class in relation to other concepts. This study tool will include functionality that allows users to separate 3D anatomical images into anatomically significant subparts, and put them back together again [Molholt et al. 1998; Wacholder et al. 1998].

- a navigational tool that allows users to locate images of interest in an application-independent collection of anatomical images, without being distracted by large numbers of irrelevant images. For this project, the collection will include images reconstructed from the Visible Human dataset, supplemented with drawings and other images as needed.

In this paper, we will discuss the Vesalius Anatomy Browser in more detail, with a focus on

1) the kinds of information that needed to be represented in a navigational ontology for anatomy;

2) the creation of the ontology by merging of information contributed by the University of Washington Digital Anatomist Project [Rosse et al. 1998] to the UMLS [Lindberg et al. 1993] with the Wacholder-Venuti ontology [Wacholder et al. under submission] and

3) the direct interaction between the user and the ontology that the Vesalius Anatomy Browser supports.

\section{Background}

The overall motivation for our research is based on the need to browse and navigate collections of anatomical images in medical education. In this section we focus on three issues:

1. the need for electronic resources to supplement anatomy education in the medical professions;

2. concept mapping;

3. representation of knowledge in anatomical ontologies.

\subsection{Anatomy information and anatomy education}

Although the fundamental importance of anatomy in medical education and clinical practice is widely recognized, it has become increasingly difficult to allocate in the medical curriculum the time and resources required for the study of anatomy [Drake 1998]. While the importance of understanding human anatomy for the practice of medicine is indisputable, the 
Wacholder, N., Venuti, J., Krauthammer, M., \& Molholt, P. (2000). Accessing and browsing 3D anatomical images with a navigational ontology..

11th ASIS SIG/CR Classification Research Workshop, 75-85. doi:10.7152/acro.v11i1.12770

net effect of these changes is that devoting classroom time to teaching all the anatomical details that first year medical students have traditionally been required to learn has become unrealistic. There is therefore an urgent need to identify new ways to convey anatomical knowledge compatible with a medical school curriculum like Columbia's (Molholt et al. 1998; Venuti 1998; Venuti 2000).

The Columbia University Department of Clinical Anatomy and Cell Biology is actively seeking ways to 'shore up' anatomy education in this new educational environment. Various alternatives to classical dissections in anatomy laboratories are being considered. Concrete physical anatomical models made from plastic or other materials are available, but they typically offer only restricted views of the anatomy. Prosections, i.e., pre-dissected regions for viewing and study, can be used to study specific anatomical relationships. However, there is some question as to the cost effectiveness of providing sufficient numbers and variations of prosections: since dissection destroys outer structures in order to reach underlying structures, distinct prosections must be generated for each level of dissection.

A better possibility is to supplement traditional teaching tools such as lectures, textbooks and cadaveric dissection with an electronic representation of anatomical information that can be flexibly reused, filtered, and expanded. State of the art 3D medical visualization allows building of sophisticated 3D environments to simulate dissection of human cadavers. Our experience has shown that electronic tools which allow anatomical structures to be deconstructed and reconstructed from interactive medical illustrations (Venuti 1998; http://cpmcnet.columbia.edu:8000/anatomy/pelvis), are useful in anatomy education and we believe that they will also be useful in other applications which require anatomical knowledge such as virtual surgery or computerized diagnosis.

Our project uses the NLM-sponsored Visible Human Dataset to reconstruct realistic 3D models of anatomical structures that can be taken apart and reconstructed in a kind of virtual anatomy. (Ackerman 1995; Spitzer 1995; Wacholder et al. 1998; Wacholder et al. (to appear)). Imielinska and others have developed techniques for reducing the size of image files produced from the Visible Human dataset enough to display these images on mid-range computers, while preserving their most important visual properties (Imielinska et al. 1998; Imielinska et al. 1996).

\subsection{Browsing and navigating anatomical images}

The potential of electronic representations of anatomical structures for education, clinical practice and research cannot be realized if images are presented in isolation. Users need to be able to locate specific images from among a potentially very large collection and view them in relation to other pertinent structures. To do this, they need to navigate from one image to another, explore relationships between structures in different images, and link structures from different regions of the body. As part of this system, users need to access images not displayed on the screen. Therefore some form of concept-based access system is required. At a minimum, images must be labeled so that they can be located and retrieved. In most cases, descriptions and explanatory material are needed to convey the significance of an anatomical image or its features. 
Wacholder, N., Venuti, J., Krauthammer, M., \& Molholt, P. (2000). Accessing and browsing 3D anatomical images with a navigational ontology..

11th ASIS SIG/CR Classification Research Workshop, 75-85. doi:10.7152/acro.v11i1.12770

The most commonly used symbolic system for accessing images is an alphabetical index organized by concept name, as in a back-of-the-book index. In printed atlases, images of anatomical structures are labeled and organized according to some classificational scheme, usually by region or system, and then listed in an alphabetical index. But as indexes grow longer, they become cumbersome. For example, a user viewing the aortic valve might also want to see the xiphoid process, but these two terms will be pretty far apart in any alphabetical list of anatomical concepts that is reasonably complete. For the thorax alone, the University of Washington Digital Anatomist Project has assigned 8700 concepts to 200 classes and subclasses [Brinkley and Rosse 1997]. The need for alphabetical indexes is unlikely to disappear, but a more sophisticated tool, organized conceptually as well as alphabetically, is required for navigating large collections of anatomical images

\subsection{Concept mapping}

Concept mapping is a technique for graphical representation of the interrelationships between concepts. Typically, concept maps consist of concepts linked by propositional relationships Edmondson (1995) Figure 2 illustrates a fragment of a concept map, adapted from Edmondson 1995.

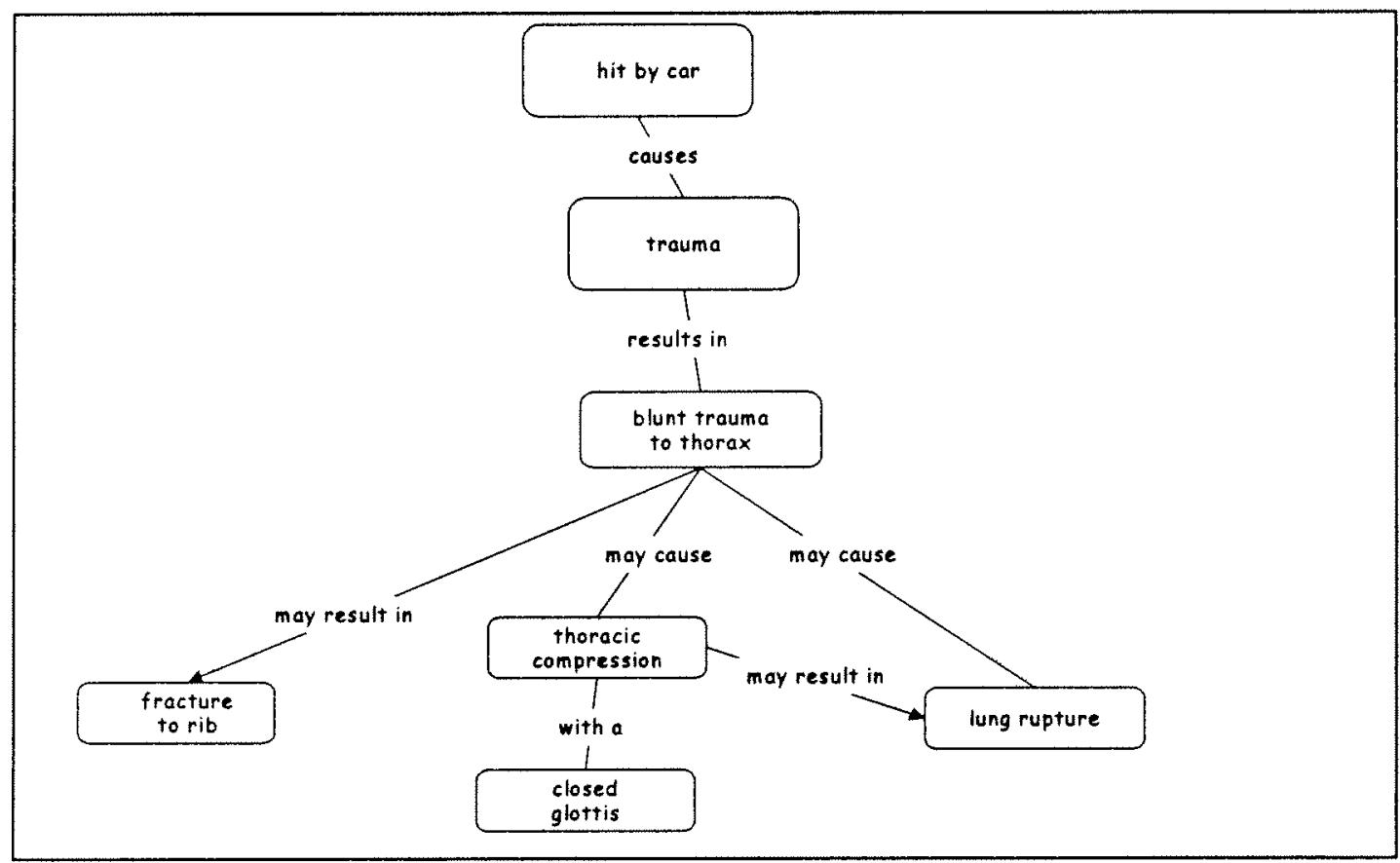

Figure 2: Fragment of a concept map, from (Edmondson 1995).

This concept map represents, for example, the proposition that blunt trauma to the thorax, caused by being hit by a car, may result in a fractured rib, in thoracic compression, or lung rupture. 
Wacholder, N., Venuti, J., Krauthammer, M., \& Molholt, P. (2000). Accessing and browsing 3D anatomical images with a navigational ontology..

11th ASIS SIG/CR Classification Research Workshop, 75-85. doi:10.7152/acro.v11i1.12770

Concept mapping is used in science education in general and medical education in particular to promote cognitive learning and assess student knowledge (Edmondson 1995; Pinto and Zeitz 1997; Jonassen et al. 1997). Concept maps promote learning by providing an explicit visual representation of conceptual information. Concept maps are valuable learning tools because they promote 'effective understanding' by emphasizing important themes, and illustrating the nature of relationships among concepts. <Edmondson and Novak 1995; Edmondson 1995 $>$. They help learners acquire a correct organization of material that may be linked to already-held knowledge and may also provide a basis for organizing subsequently acquired knowledge (Novak 1990).

Concept maps are usually developed informally, with no plan for consistency of concepts across maps. Typically they are small, usually less then 100 concepts (Jonassen et al. 1997). Concepts are not systematically classified by semantic class: in figure 2 , an event ("hit by car") causes a physical consequence ("trauma"), which results in a medical emergency ("blunt trauma to thorax"). There is no systematic limitation on the propositional relationships that may be used in the concept map: relationships are drawn from spoken language as needed to represent information. For example, in Figure 2, the relationships include cause, result, and with. Concept maps can be used to illustrate any concept and any relationship, thereby making them useful for any domain or field of study. Because they are frequently used to represent the knowledge of individuals, concept maps are not typically associated with a notion of standardization, where the representation in the concept map is systematic and therefore relatively easily shared between individuals. This flexibility is an advantage for representing the knowledge of an individual but a disadvantage for the systematic transfer of knowledge between individuals.

In the next section, we analyze semantic ontologies as a kind of concept map, with formal restrictions on their organization that on the one hand restrict the concept-mapper's choice of relationships and concepts, but on the other hand produce representations with consistent relationships among concepts in different parts of the ontology.

\section{Semantic ontologies}

An ontology is a type of knowledge representation system which includes common sense knowledge organized so that a computer system can perform deductive reasoning. An ontology consists of a set of terms, belonging to some domain of interest, associated with definitions in natural language; ontologies are used by people, databases and computer programs. Ontologies are like concept maps in that they formally represent relationships among concepts, but differ from concept maps in the concepts are strictly classified and relationships formally defined. 
Wacholder, N., Venuti, J., Krauthammer, M., \& Molholt, P. (2000). Accessing and browsing 3D anatomical images with a navigational ontology..

11th ASIS SIG/CR Classification Research Workshop, 75-85. doi:10.7152/acro.v11i1.12770

The conceptual information in the display of the VAB in Figure 1 is shown graphically in Figure 3

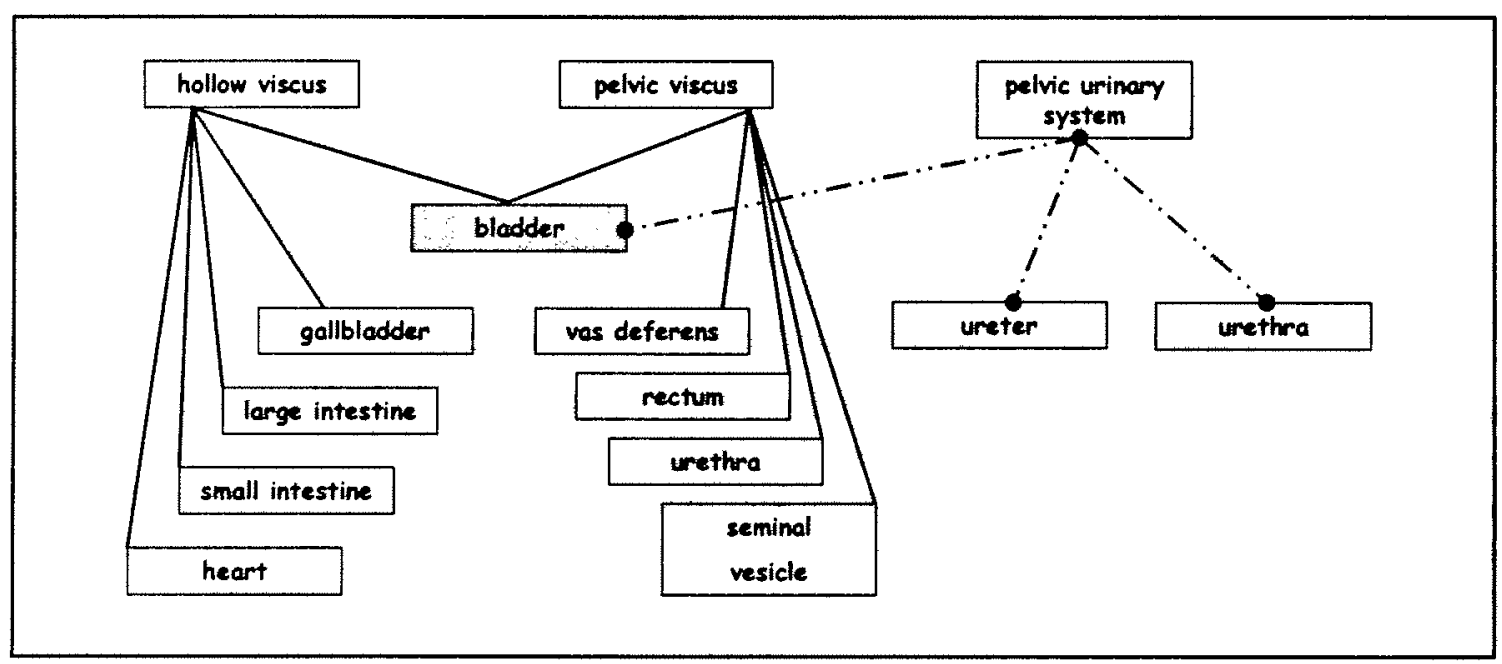

Figure 3: Concept space for bladder

The VAB produces the text-based display in Figure 1 instead of the graphical display in Figure 3 because it does not yet have the capability in this graphically complex format.

The fragment of the ontology in Figure 3 uses two systematically defined relationships: the is-a relationship and the component-structure relationship.

- The is-a relationship The formal relationship most widely used in ontologies in all domains is generally referred to as the taxonomic or is-a relation, a definitional relationship between a general class (e.g., birds) and a more specific set of subclasses (e.g., canaries and penguins). In the is-a relation, each subclass shares (technically, 'inherits') all of the properties of the more general class (i.e., all classes of birds have feathers, two legs and two wings) and is also characterized by additional properties (differentia) that distinguish it from other subclasses (e.g., canaries are yellow and can fly; penguins are black and white and cannot fly). The $i s-a$ relationship has been extensively studied and its properties are relatively well understood (Brachman 1992; Guarino 1998; Wacholder (to appear)). 


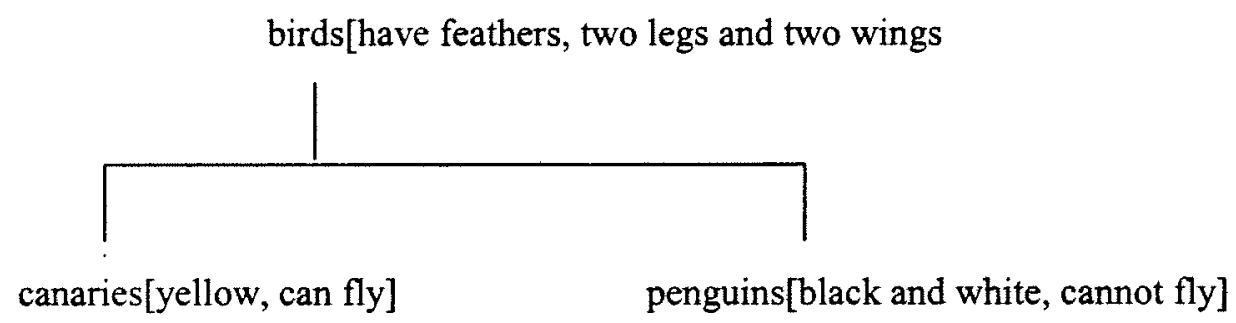

Figure 4. Definitional/classificatory relationship (is-a)

The component-structure relationship is much like what Winston et al. [1987] termed 'component-integral object' relationship, where "an 'integral object' is divided into components...that usually bear specific structural and functional relationships to one another and to the wholes which they compose". The component-structure relationship is illustrated in Fig. 5, where $\mathbf{S}$ is an abstract representation of some concrete anatomical structure.

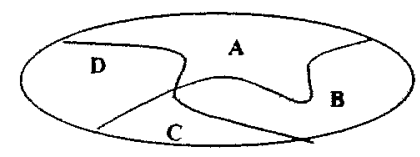

Figure 5: $\langle\mathrm{A}, \mathrm{B}, \mathrm{C}, \mathrm{D}]$ component-structures- of $\mathrm{S}\rangle$

The lines separating the components represent borders which are either visible to the naked eye or can be discovered on close physical examination. $\mathbf{S}$ can be subdivided into four independent structures, $\mathbf{A}, \mathbf{B}, \mathbf{C}$ and $\mathbf{D}$, and recombined only in the configuration shown in Fig. 3 to reconstruct $\mathbf{S}$. For example, the pelvic urinary system consists of three components: the bladder, the ureter, and the urethra. In general, part-whole relations are less well understood than $i s-a$ relations (Artale et al. 1996).

Because they require formal definition of semantic classes and relationships, semantic ontologies require considerably more effort to create than do concept maps. However, the payoff is that semantically ontologies, formally defined in terms of inheritance properties by which classes are related, can be used to support deductive reasoning about anatomy by a computer system. To give a simple example, if a prostate is- $\boldsymbol{a}$ viscus and a viscus is- $\boldsymbol{a}$ body organ, then a computer system can be programmed to deduce that the prostate is a body organ, and the prostate can be listed or displayed whenever a user asks for a list of body organs

\section{Creating a navigational ontology}

Creation of an anatomical ontology is an enormous task that can only be accomplished through knowledge sharing and re-use (Musen 1992; Rector 1999; Krauthammer 1999). To create the Vesalius Navigational Ontology, we therefore turned to the University of Washington Digital Anatomist Ontology (UWDA), which was designed as an applicationindependen ${ }^{+}$ontology and contributed to the UMLS. To facilitate successful re-use of the 
Wacholder, N., Venuti, J., Krauthammer, M., \& Molholt, P. (2000). Accessing and browsing 3D anatomical images with a navigational ontology..

11th ASIS SIG/CR Classification Research Workshop, 75-85. doi:10.7152/acro.v11i1.12770

UWDA ontology, we have collaborated with the UWDA, whose members have generously shared with us aspects of their reasoning, motivation and work-in-progress. Most of the is-a relationships in our navigational ontology were taken from the UWDA ontology, as contributed to the UMLS (NLM 1999).

The UWDA ontology as available in the 1999 edition of the UMLS Knowledge Sources, although it includes part-whole relationships, does not include this information with the specificity needed for intelligent navigation. We therefore merged it with the WacholderVenuti ontology, developed locally for this purpose. The merging process was accomplished automatically with the MEDEditor Suite as described in (Krauthammer 1999 and Krauthammer et al. (to appear)). We successfully merged the UWDA ontology with the Wacholder-Venuti ontology into the Vesalius Knowledge Base, which is accessed by the Vesalius Anatomy Browser.

\section{Concept maps and image access}

The primary reason for use of the semantic ontology to access anatomical images is that the relationships between anatomical concepts also hold for the links between images associated with these concepts. In our navigational ontology, we take advantage of these associative relationships by linking $3 \mathrm{D}$ anatomical images with a piece of an ontology which indicates the relationship of other anatomical concepts to the focus concept. The underlying logic behind the display is that a user interested in looking a given anatomical entity is likely to want to view next an anatomical structure related to it in some significant way. By bringing together in the concept space anatomical concepts that are linked by meaningful relations and by linking anatomical concepts to anatomical images, the ontology effectively functions as a context-sensitive navigational system.

The display of the image and the corresponding view of the concept space emphasize the anatomical entity's relations to related entities. The navigational ontology is a useful learning tool for the same reasons that a concept map is, and it is reinforced by its use in a domain where concepts are associated with specific images, so that the concept space reinforces the representation of the images, and provides a context for them.

The navigational ontology retains the important advantage of concept maps: it explicitly displays important relationships between concepts in a way that provides to the user contextual important.

The association of the concepts with images makes possible simultaneous visual exploration via word and image. 
Wacholder, N., Venuti, J., Krauthammer, M., \& Molholt, P. (2000). Accessing and browsing 3D anatomical images with a navigational ontology..

11th ASIS SIG/CR Classification Research Workshop, 75-85. doi:10.7152/acro.v11i1.12770

84 Wacholder et al., Accessing and Browsing 3D Anatomical Images with a Navigational Ontology

\section{References}

Ackerman, Michael J. (1995) "Fact Sheet: The Visible Human Project", National Library of Medicine.

Artale, Alessandro, Enrico Franconi, and Nicola Guarino (1996) "Open problems with partwhole relations", International Workshop on Description Logics, Boston, MA, 1996.

Brachman, Ronald J. (1982) "What IS-A is and isn't: an analysis of taxonomic links in semantic networks", Proceedings of Fourth National Conference of the Canadian Society for Computational Studies of Intelligence, May, 1982, IEEE.

Brinkley, James and Cornelius Rosse (1997) "The Digital Anatomist distributed framework and its applications to knowledge-based medical imaging". JAMIA 4(165-183).

Drake, Richard L. (1998) "Anatomy education in a changing medical curriculum", New Anatomist 243:28-31.

Edmondson, Katherine M. "Concept mapping for the development of medical curricula", Journal of Research in Science Teaching 32(7):777-793.

Guarino, Nicola (1998) "Some ontological principles for designing upper level lexical resources". Proceedings of the First International Conference on Language Resources and Evaluation, pp.527-534. Grenada, Spain, May 28-20, 1998.

Horton, P. B., A. A. McConney, M. Gallo, A. L. Woods, G. J. Senn and Denis Hameln (1993) "An investigation of the effectiveness of concept mapping as an instructional tool", Science Education 77(1):95-111.

Imielinska, Celina., Lisa Laino-Pepper, Roger Thumann and Ryan Villamil (1998) "Technical Challenges of 3D Visualization of Large Color Data Sets", Proceedings of Second User Conference of the National Library of Medicine's Visible Human Project, October 1, 1998, Bethesda, MD.

Imielinska, C., M. Downes, S. Hosakere, A. Khan and W. Yuan (1996) W., "2D Segmentation of Color Anatomical Data". Proceedings of the First User Conference of the National Library of Medicine's Visible Human Project, October 8-9th, 1996, Bethesda, MD; also accepted to a special issue of the Journal of Computerized Medical Imaging and Graphics.

Jonassen, David H., Thomas C. Reeves, Namsoo Hong, Douglas Harvey and Karen Peters (1997) "Concept mapping as cognitive learning and assessment tools" Journal of Interactive Learning Research 8(3/4):289-308.

Krauthammer, Michael (1999) "Implementing the Vesalius Anatomy Browser", Unpublished Master's Thesis, Columbia University, New York.

Lindberg, Diane A, Betsy L. Humphreys and Alexa T. McCray (1993) "The Unified Medical Language System" Methods Inf Med. 32(4):281-91.

Molholt Pat, Celina Imielinska, Judith Klavans, Lisa Laino-Pepper, Ewa Soliz and Hilary Schmidt (1998) "The Vesalius Project: Creating a Computer Based Anatomy Curriculum" Proceedings of the Second User Conference of the National Library of Medicine's Visible Human Project, Bethesda, MD.

Musen, Mark (1992) "Dimensions of knowledge sharing and reuse" Computers and Biomedical Research 25:435-467.

Novak, J. D. (1990) “Concept mapping: a useful tool for science education”, Journal of Research in Science Teaching 27(10):937-949.Educational Research Journal. 
Wacholder, N., Venuti, J., Krauthammer, M., \& Molholt, P. (2000). Accessing and browsing 3D anatomical images with a navigational ontology..

11th ASIS SIG/CR Classification Research Workshop, 75-85. doi:10.7152/acro.v11i1.12770

Wacholder et al., Accessing and Browsing 3D Anatomical Images with a Navigational Ontology

Pinto, Angelo J. and Howard J. Zeitz (1997) "Concept mapping: a strategy for promoting meaningful learning in medical education", Medical Teacher 19(2):114-121.

Rector, A. L. et al. (1999) "Reconciling user's needs and formal requirements: issues in developing a re-usable ontology for medicine", IEEE Transactions 2(4):229-242.

Rosse, C., J. L. Mejino, B. R. Modayur, R. Jakobovits, K. P. Hinshaw, and J. F. Brinkley (1998) "Motivation and organizational principles for anatomical knowledge representation", JAMIA $5(1): 17-40$.

Spitzer, V. et al. "The Visible Human Male: A technical report", JAMIA, 1995.

Venuti, J., Soliz, E., Imielinska, C., Molholt, P., Wacholder, N., "Development of a Pelvic Anatomy Lesson: Innovation in Electronic Curriculum for Medical Education", Proceedings of the Second User Conference of the National Library of Medicine's Visible Human Project, October 1, 1998, Bethesda, MD.

Wacholder Nina, J. Venuti, M. Krauthammer and P. Molholt (to appear) "Part-whole relations in a navigational ontology for browsing and accessing 3D anatomical images", Proceedings of the Third Visible Human Conference.

Wacholder, Nina, Celina Imielinska, Judith Klavans, and Pat Molholt (1998) "Semantic relations in a medical digital library", Proceedings of the American Digital Library Conference, Santa Barbara, CA, April 1998.

Wacholder, Nina, Celina Imielinska, C., Judith Klavans and Pat Molholt. P. (1998) "Navigating 3D Anatomical Images with a Semantic Ontology", Proceedings of the Second User Conference of the National Library of Medicine's Visible Human Project, October 1, 1998, Bethesda, MD. 